\title{
RIGIDITY OF VECTOR VALUED HARMONIC MAPS OF LINEAR GROWTH
}

\author{
SHAOSAI HUANG AND BING WANG
}

\begin{abstract}
Consider vector valued harmonic maps of at most linear growth, defined on a complete non-compact Riemannian manifold with non-negative Ricci curvature. For the norm square of the pull-back of the target volume form by such maps, we report a strong maximum principle, and equalities among its supremum, its asymptotic average, and its large-time heat evolution.
\end{abstract}

\section{INTRODUCTION}

We fix a complete, non-compact Riemannian manifold $(M, g)$ with non-negative Ricci curvature. Harmonic functions on such manifolds have been an important subject of investigation in geometric analysis (see, among others, [24], [14], [15], [18], [13], [16], [17], [10], [11], and [6], etc.).

Among all harmonic functions, those of at most linear growth are especially interesting, as they reveal the properties of the tangent cones at infinity of $M$, through the work of Cheeger-Colding-Minicozzi II [6]. Vector valued harmonic maps of at most linear growth are also of central imporance in studying the structure of the Gromov-Hausdorff limits of a sequence of Riemannian manifolds with Ricci curvature uniformly bounded below, as signified in the series of work by Cheeger-Colding (see [2], [3], [4], and [5]), and notably the recent resolution of the codimension- 4 conjecture by Cheeger-Naber [8].

Our subject of study is a vector valued harmonic map of at most linear growth. To fix the terminologies, we call a function $\mathbf{u}:\left(M^{m}, x_{0}\right) \rightarrow\left(\mathbb{R}^{n}, \mathbf{0}\right)(n \leq m)$ a vector valued harmonic map if each component of $\mathbf{u}$ is a harmonic function $u_{\alpha}: M \rightarrow \mathbb{R}(\alpha=1, \cdots, n)$, and call it pointed if $\mathbf{u}\left(x_{0}\right)=\mathbf{0}$. Moreover, we say that $\mathbf{u}$ is of at most linear growth if there exists some $L>0$, such that

$$
\forall x \in M, \quad\left|\mathbf{u}(x)-\mathbf{u}\left(x_{0}\right)\right| \leq L(r(x)+1),
$$

where $\forall x \in M, r(x):=d\left(x, x_{0}\right)$, the geodesic distance between $x$ and $x_{0}$, induced by the Riemannian metric $g$.

To each vector valued harmonic map as above, we could associate an $n$-form

$$
\omega:=\mathbf{u}^{*}\left(d y^{1} \wedge d y^{2} \wedge \cdots \wedge d y^{n}\right) \in \Gamma\left(M, \Lambda^{n} T^{*} M\right) .
$$

We say that $\mathbf{u}$ is non-trivial if $|\omega| \not \equiv 0$.

For any $A \in G L(n)$, define $\omega_{A}:=(A \mathbf{u})^{*}\left(d y^{1} \wedge d y^{2} \wedge \cdots \wedge d y^{n}\right)$. The following invariance of the pull-back $n$-form under the induced $S L(n)$-actions is fundamental for our arguments:

$$
\forall A \in S L(n), \quad\left|\omega_{A}\right|^{2}=\operatorname{det} A|\omega|^{2}=|\omega|^{2} .
$$

Our first result then states:

Date: January 10, 2018.

Key words and phrases. Harmonic map, Heat kernel, Ricci curvature.

Both authors are partially supported by NSF grant DMS-1510401. 
Theorem 1.1 (Strong maximum principle). Let $\mathbf{u}:\left(M, x_{0}\right) \rightarrow\left(\mathbb{R}^{n}, \mathbf{0}\right)$ be a pointed, vector valued harmonic map of at most linear growth which is non-trivial at $x_{0}$. Then

$$
\lim _{\rho \rightarrow \infty} f_{B\left(x_{0}, \rho\right)}|\omega|^{2} d V_{g}=\sup _{M}|\omega|^{2} .
$$

Moreover, if $|\omega|\left(x_{0}\right)=\sup _{M}|\omega|$, then $|\nabla \nabla \mathbf{u}| \equiv 0$, and $\left(M^{m}, g\right) \equiv\left(N^{m-n}, h\right) \times\left(\mathbb{R}^{n}, g_{\text {Euc }}\right)$ isometrically, with $\left(N^{m-n}, h\right)$ being some $(m-n)$-dimensional Riemannian manifold with non-negative Ricci curvature.

Clearly, there is nothing special about the choice of base point $x_{0}$ in the statement of the theorem. Our proof of this theorem is a blend of the heat kernel estimates due to Li-Yau (see [20] and [14]), and the Hessian $L^{2}$-estimates by Cheeger-Colding-Minicozzi II [6]. Now we briefly discuss the ingredients involved in Theorem 1.1 .

Notice, since $\mathcal{R} c \geq 0$, that each individual $\left|\nabla u_{\alpha}\right|^{2}(\alpha=1, \cdots, n)$ is sub-harmonic by the Weitzenböck formula:

$$
\Delta\left|\nabla u_{\alpha}\right|^{2}=2\left|\nabla \nabla u_{\alpha}\right|^{2}+2 \mathcal{R} c\left(\nabla u_{\alpha}, \nabla u_{\alpha}\right) \geq 0,
$$

also notice that the linear growth of $u_{\alpha}$ gives a global upper bound of $\left|\nabla u_{\alpha}\right|^{2}$ by the ChengYau gradient estimate [9]: $\left|\nabla u_{\alpha}\right|^{2}$ is a bounded, non-negative sub-harmonic function on $M$. By a classical theorem of Peter Li [15], we have

$$
\lim _{\rho \rightarrow \infty} f_{B\left(x_{0}, \rho\right)}\left|\nabla u_{\alpha}\right|^{2} \mathrm{~d} V_{g}=\sup _{M}\left|\nabla u_{\alpha}\right|^{2} .
$$

Therefore we could view (1.2) as a high-dimensional generalization of this identity for the energy of harmonic functions of at most linear growth.

The proof of (1.2) is based on the invariance of certain canonical quantities associated to a vector valued harmonic map $\mathbf{u}$, and the invariance properties enable the compactness of $S O(n)$ to work for limiting arguments. Besides the pull-back measure density of $\mathbf{u}$, we also define:

(1) Energy density: $|\nabla \mathbf{u}|^{2}:=\sum_{\alpha=1}^{n}\left|\nabla u_{\alpha}\right|^{2}$;

(2) Splitting error: $r^{2}|\nabla \nabla \mathbf{u}|^{2}:=\sum_{\alpha=1}^{n} r^{2}\left|\nabla \nabla u_{\alpha}\right|^{2}$.

We notice that the quantities $|\omega|^{2},|\nabla \mathbf{u}|^{2}$ and $r^{2}|\nabla \nabla \mathbf{u}|^{2}$ are invariant under the special orthogonal group actions on $\mathbb{R}^{n}$ :

$$
\forall A \in S O(n), \quad \mathbf{u} \mapsto A \mathbf{u}, \quad \text { i.e. }(A \mathbf{u})_{\alpha}:=\sum_{\beta=1}^{n} A_{\alpha \beta} u_{\beta} .
$$

For the rigidity part of Theorem 1.1, i.e. when $|\omega|\left(x_{0}\right)=\sup _{M}|\omega|$ happens, we observe that (1.3) links the difference of the average energy between two scales, and this observation is implemented through the application of the heat measure. Here the heat measure is defined as $\mathrm{d} \mu_{x_{0}}(t):=H_{x_{0}}(\cdot, t) \mathrm{d} V_{g}$, where $H_{x_{0}}(y, t)$ is the fundamental solution to the heat equation (in $y \in M$ ) with the Delta function at $x_{0} \in M$ as its initial value. Summing (1.3) in $\alpha=1, \cdots, n$, the aforementioned observation could be expressed as (see [14] for the justification of integration by parts)

$$
\frac{\mathrm{d}}{\mathrm{d} t} \int_{M}|\nabla \mathbf{u}|^{2} \mathrm{~d} \mu_{x_{0}}(t) \geq 2 \int_{M}|\nabla \nabla \mathbf{u}|^{2} \mathrm{~d} \mu_{x_{0}}(t),
$$

so integrating between different scales $\rho_{1}<\rho_{2}$, we get

$$
\int_{M}|\nabla \mathbf{u}|^{2} \mathrm{~d} \mu_{x_{0}}\left(\rho_{2}^{2}\right)-\int_{M}|\nabla \mathbf{u}|^{2} \mathrm{~d} \mu_{x_{0}}\left(\rho_{1}^{2}\right) \geq 2 \int_{\rho_{1}^{2}}^{\rho_{2}^{2}} \int_{M}|\nabla \nabla \mathbf{u}|^{2} \mathrm{~d} \mu_{x_{0}}(t) \mathrm{d} t .
$$


This inequality contains rich information about $\mathbf{u}$ : it tells not only that the weighted energy (weighted by the heat measure based at $x_{0} \in M$ ) on large scales dominates that on smaller ones, but also that the difference dominates the splitting error. Roughly speaking, if $|\omega|^{2}$ attains a global maximum at $x_{0}$, this inequality then forces the splitting error to vanish.

Following a similar argument and with the help of a Poincaré inequality weighted by the heat measure (Lemma4.1), we also prove:

Theorem 1.2 (Large-time heat evolution). Let $\left(M, g, x_{0}\right)$ and $\mathbf{u}$ satisfy the assumption of Theorem 1.1 then

$$
\lim _{t \rightarrow \infty} \int_{M}|\omega|^{2} d \mu_{x_{0}}(t)=\sup _{M}|\omega|^{2}
$$

This theorem, in conjunction with Theorem 1.1 then says

$$
\lim _{\rho \rightarrow \infty} f_{B\left(x_{0}, \rho\right)}|\omega|^{2} \mathrm{~d} V_{g}=\sup _{M}|\omega|^{2}=\lim _{t \rightarrow \infty} \int_{M}|\omega|^{2} \mathrm{~d} \mu_{x_{0}}(t) .
$$

Notice that $|\omega|^{2}$ is not necessarily sub-harmonic in any obvious way (see Section 4 for more details), therefore we have to prove both sides of (1.8) separately. At this point, it is interesting to compare this identity with classical results of Peter Li in [15]:

Theorem 1.3 (Peter Li [15]). Suppose $M$ has positive asymptotic volume ratio at infinity, i.e. $\lim _{\rho \rightarrow \infty} \omega_{m}^{-1} \rho^{-m} \operatorname{Vol}_{g}\left(B\left(x_{0}, \rho\right)\right)=\kappa>0$, then for any bounded function $f$,

$$
\lim _{\rho \rightarrow \infty} f_{B\left(x_{0}, \rho\right)} f d V_{g}=\lim _{t \rightarrow \infty} \int_{M} f d \mu_{x_{0}}(t),
$$

as long as one of these limits exists.

On the other hand, if $f$ is a bounded, non-negative sub-harmonic function, then regardless of the positivity of the asymptotic volume ratio at infinity,

$$
\lim _{\rho \rightarrow \infty} f_{B\left(x_{0}, \rho\right)} f d V_{g}=\sup _{M} f \text { and } \lim _{t \rightarrow \infty} \int_{M} f d \mu_{x_{0}}(t)=\sup _{M} f .
$$

Notice that when $M$ has vanishing asymptotic volume ratio at infinity, (1.9) is not necessarily true for any bounded function, see [23]. However it is not clear if (1.10) only holds for sub-harmonic functions. Therefore, (1.8) provides another incidence when (1.10) stands for a function which is not necessarily sub-harmonic, regardless of the positivity of the asymptotic volume ratio at infinity. See Section 4 for a further discussion.

\section{BACKGROUND}

In this section we review the relevant facts needed for our future arguments: CheegerColding's segment inequality, and the Li-Yau heat kernel estimates. We will also recall a result due Peter $\mathrm{Li}$, the proof of which was embedded in other results and here we single it out. Instead of citing the original results in full generality, we will state the results in a form that suit our future applications.

Given any Riemannian manifold with Ricci curvature uniformly bounded below, a fundamental inequality which is directly built on the Bishop-Gromov volume comparison is Cheeger-Colding's segment inequality [2] (see also [1]): 
Proposition 2.1 (Segment inequality). Let $\left(M^{m}, g\right)$ be a complete Riemannian manifold with $\mathcal{R} c_{g} \geq 0 \mathrm{~g}$. Let $B\left(x_{0}, r\right)$ be a geodesic ball of radius $\rho>0$ around $x_{0} \in M$. For any $f \in L_{\text {loc }}^{1}(M)$ we define

$$
\mathcal{F}(x, y):=\inf _{\gamma_{x y}} \int_{0}^{d(x, y)} f\left(\gamma_{x y}(t)\right) d t,
$$

where the infimum is taken over all minimal geodesics $\gamma_{x y}$ connecting $x$ and $y$. There is a dimensional constant $C_{C C}(m)>0$ such that for any $f \in L_{l o c}^{1}(M)$,

$$
\int_{B\left(x_{0}, \rho\right) \times B\left(x_{0}, \rho\right)} \mathcal{F}(x, y) d V_{g}(x) d V_{g}(y) \leq C_{C C}(m)\left|B\left(x_{0}, \rho\right)\right|(2 \rho) \int_{B\left(x_{0}, 2 \rho\right)} f d V_{g} .
$$

This inequality is useful in extracting estimates along most geodesics connecting pairs of points, see for instance [2] and [12]. It also has the Poincaré inequality as a natural consequence (see [21] and [1]):

Proposition 2.2 (Poincaré inequality). Assume $(M, g)$ is a complete Riemannian manifold with non-negative Ricci curvature. There is a dimensional constant $C_{P}(m)>0$ such that for any $f \in W_{l o c}^{1,1}(M)$,

$$
f_{B\left(x_{0}, \rho\right)}\left|f-f_{B\left(x_{0}, \rho\right)} f d V_{g}\right| d V_{g} \leq C_{P}(m) \rho f_{B\left(x_{0}, 2 \rho\right)}|\nabla f| d V_{g} .
$$

In fact, with the help of the segment inequality, we will later prove a version of the Poincaré inequality (Lemma 4.1) with the heat measure $\mathrm{d} \mu_{x_{0}}$ replacing the volume form $\mathrm{d} V_{g}$. To set up the weighted Poincare inequality, the heat kernel estimates due to LiYau [20] is also of crucial importance:

Proposition 2.3 (Heat kernel bounds). Let $(M, g)$ be a complete, non-compact Riemannian manifold with non-negative Ricci curvature. Then the fundamental solution $H_{x_{0}}(x, t)$ to the heat equation satisfies

$$
\frac{C_{1}(\varepsilon)^{-1}}{\left|B\left(x_{0}, \sqrt{t}\right)\right|} e^{-\frac{r^{2}(x)}{(4-\varepsilon) t}} \leq H_{x_{0}}(x, t) \leq \frac{C_{2}(\varepsilon)}{\left|B\left(x_{0}, \sqrt{t}\right)\right|} e^{-\frac{r^{2}(x)}{(4+\varepsilon) t}}
$$

where the positive constants $C_{1}(\varepsilon), C_{2}(\varepsilon) \rightarrow \infty$ as $\varepsilon \rightarrow 0$.

Moreover, we will need the following Harnack inequality for heat kernels, which is also proved in [20]:

Proposition 2.4 (Harnack inequality for positive heat equation solutions). Let $w(x, t)$ be a positive solution to the heat equation, then for $x, y$ and $t_{1}<t_{2}$ we have

$$
w\left(x, t_{1}\right) \leq w\left(y, t_{2}\right)\left(\frac{t_{2}}{t_{1}}\right)^{\frac{3 m}{4}} \exp \left(\frac{3 d(x, y)^{2}}{8\left(t_{2}-t_{1}\right)}\right) .
$$

As a consequence of the above Harnack inequality, we have the following lemma due to Peter Li [15], see the second identity of [1.10]:

Lemma 2.5. Let u be a bounded, non-negative sub-harmonic function defined on a complete non-compact Riemannian manifold $(M, g)$ with non-negative Ricci curvature. Let $d \mu_{x}$ be the heat measure based at $x \in M$, then

$$
\lim _{t \rightarrow \infty} \int_{M} u d \mu_{x}(t)=\sup _{M} u
$$


The proof of this lemma is contained in the proof of Proposition 2 in [15]. We include it here for the sake of completeness.

Proof. For any $x \in M$ and $t>0$ we define $H u(x, t):=\int_{M} u \mathrm{~d} \mu_{x}(t)$. By the sub-harmonicity of $u$ we see that $H u(x, t)$ is non-decreasing in $t$ :

$$
\forall x \in M, \forall t>0, \quad \partial_{t} H u(x, t)=\int_{M} \Delta u \mathrm{~d} \mu_{x}(t) \geq 0 .
$$

Especially $H u(x, t) \geq u(x)$ for any $x \in M$ and any $t>0$. Moreover, the positivity of $H_{x}(y, t)$ (the fundamental solution to the heat equation based at $x \in M$ ) and the stochastic completeness of $\mathrm{d} \mu_{x}(t)$ ensure that

$$
\forall x \in M, \forall t>0, \quad 0 \leq H u(x, t) \leq \sup _{M} u .
$$

With the help of (2.3), we see that as $t \rightarrow \infty, H u(-, t)$ converges to a function uniformly on compact subsets of $M$. Choosing a compact exhaustion of $M$ and using a diagonal argument of choosing sub-sequences, we conclude that as $t \rightarrow \infty, H u(-, t)$ converges to some globally defined function $H u(-, \infty)$, uniformly on compact subsets of $M$.

Clearly $0 \leq H u(-, \infty) \leq \sup _{M} u$, and in fact $H u(-, \infty)$ is harmonic. Therefore, by the Cheng-Yau gradient estimate [9], $H u(-, \infty)$ must be a constant, which must be $\sup _{M} u$, since $H(x, \infty) \geq u(x)$ for any $x \in M$.

\section{The STRONG MAXIMUM PRINCIPLE}

In this section we prove the strong maximum principle, Theorem 1.1 Consider the functions $E_{\alpha \beta}(\mathbf{u}):=\left\langle\nabla u_{\alpha}, \nabla u_{\beta}\right\rangle$ on $M(\alpha, \beta=1, \cdots, n)$, and the $n \times n$-matrix valued function $\mathbb{E}(\mathbf{u}):=\left[E_{\alpha \beta}(\mathbf{u})\right]$, which is positive semi-definite throughout $M$. For each $\rho>0$, we could also consider the average of $\mathbb{E}(\mathbf{u})$ over $B\left(x_{0}, \rho\right)$, a positive semi-definite numerical matrix:

$$
\Omega_{\rho}(\mathbf{u}):=\left[f_{B\left(x_{0}, \rho\right)} E_{\alpha \beta}(\mathbf{u}) \mathrm{d} V_{g}\right]
$$

Since $|\omega|^{2}=\operatorname{det} \mathbb{E}(\mathbf{u})$, the theme of the proof is the interplay between the quantities $f_{B\left(x_{0}, \rho\right)} \operatorname{det} \mathbb{E}(\mathbf{u}) \mathrm{d} V_{g}$ and $\operatorname{det} \Omega_{\rho}(\mathbf{u})$, which are obtained by taking determinant and then average of $\mathbb{E}(\mathbf{u})$, or in the alternative order.

Pick a sequence of scales $\rho_{i}$ that tend to infinity. By Li's identity (1.4), the adjusted harmonic maps (adjustments made so that $\Omega_{\rho_{i}}$ becomes diagonal) will see $\operatorname{det} \Omega_{\rho_{i}}$ approaching $\sup _{M}|\omega|^{2}$, since det $\Omega_{\rho_{i}}$ and $|\omega|^{2}$ are invariant under the induced $S O(n)$-actions, and the diagonalization enables us to deal with the determinants in a similar way as scalar functions.

On the other hand, following an argument in [6], the $L^{2}$-average of Hessian could be shown to approach zero, therefore, by the Poincaré inequality (2.1), the process of taking determinant and taking average of $\mathbb{E}(\mathbf{u})$ on larger and larger scales will gradually commute.

The last piece is the compactness of $S O(n)$, from which we could obtain certain limiting adjustment by a special orthogonal matrix that works for the asymptotic behavior.

The proof of the rigidity part follows from a direct application of the heat measure, as outlined in the introduction. We also need Lemma 2.5 to take care of individual component functions. 
Proof of Theorem 1.1 Asymptotic maximality. To prove [1.2], notice that it suffices to show the following:

$$
\forall x \in M, \quad|\omega|^{2}(x) \leq \lim _{\rho \rightarrow \infty} f_{B\left(x_{0}, \rho\right)}|\omega|^{2} \mathrm{~d} V_{g}
$$

since $f_{B\left(x_{0}, \rho\right)}|\omega|^{2} \mathrm{~d} V_{g} \leq \sup _{M}|\omega|^{2}$ for any $\rho>0$. To prove 3.1), pick any sequence $\rho_{i} \rightarrow \infty$ and let $A_{i} \in S O(n)$ diagonalize $\Omega_{\rho_{i}}(\mathbf{u})$. Then by the compactness of $S O(n)$, possibly passing to a subsequence, $A_{i} \rightarrow A_{\infty} \in S O(n)$. Denoting $\mathbf{v}:=A_{\infty} \mathbf{u}$, we have, according to (1.1) and (1.5),

$$
\begin{aligned}
& \operatorname{det} \Omega_{\rho_{i}}(\mathbf{u})=\operatorname{det} \Omega_{\rho_{i}}\left(A_{i} \mathbf{u}\right)=\operatorname{det} \Omega_{\rho_{i}}(\mathbf{v}), \\
& \text { and }|\omega|^{2} \equiv\left|\omega_{A_{i}}\right|^{2} \equiv\left|\omega_{A_{\infty}}\right|^{2} \text { on } M .
\end{aligned}
$$

Moreover, for $\lambda_{\alpha, i}^{2}:=f_{B\left(x_{0}, \rho_{i}\right)}\left|\nabla v_{\alpha}\right|^{2} \mathrm{~d} V_{g}$, we have, by the convergence $A_{i} \rightarrow A_{\infty}$, that

$$
\lim _{i \rightarrow \infty}\left|\prod_{\alpha=1}^{n} \lambda_{\alpha, i}^{2}-\operatorname{det} \Omega_{\rho_{i}}(\mathbf{u})\right|=0 .
$$

Since $A_{\infty}$ is a linear transformation, each component function of $\mathbf{v}=A_{\infty} \mathbf{u}$ is harmonic, and applying (1.4) we have, for each $\alpha=1, \cdots, n$,

$$
\lim _{i \rightarrow \infty} \lambda_{\alpha, i}^{2}=\lim _{\rho \rightarrow \infty} f_{B\left(x_{0}, \rho\right)}\left|\nabla v_{\alpha}\right|^{2} \mathrm{~d} V_{g}=\sup _{M}\left|\nabla v_{\alpha}\right|^{2}=: L_{\alpha}^{2} .
$$

Now we follow an argument in [6] to control the average Hessian of each $v_{\alpha}$ on large enough scales. For any fixed $\rho_{i}>0$, let $\varphi_{i}$ be a cutoff function defined as in [22], such that $\operatorname{supp} \varphi_{i} \subset B\left(x_{0}, 3 \rho_{i}\right)$ and $\varphi_{i} \equiv 1$ on $B\left(x_{0}, 2 \rho_{i}\right)$, moreover,

$$
\rho_{i}^{2}\left|\Delta \varphi_{i}\right|+\rho_{i}\left|\nabla \varphi_{i}\right| \leq C(M) .
$$

We can then estimate the average Hessian on scale $\rho_{i}$ :

$$
\begin{aligned}
f_{B\left(x_{0}, 2 \rho_{i}\right)} 2\left|\nabla \nabla v_{\alpha}\right|^{2} \mathrm{~d} V_{g} & \leq 2^{m} f_{B\left(x_{0}, 3 \rho_{i}\right)} \varphi_{i} \Delta\left(\left|\nabla v_{\alpha}\right|^{2}-L_{\alpha}^{2}\right) \mathrm{d} V_{g} \\
& \leq 2^{m} f_{B\left(x_{0}, 3 \rho_{i}\right)}\left|\Delta \varphi_{i}\right|\left(L_{\alpha}^{2}-\left|\nabla v_{\alpha}\right|^{2}\right) \mathrm{d} V_{g} \\
& \leq C(M) \rho_{i}^{-2} L_{\alpha}^{2} \Psi\left(\rho_{i}^{-1}\right),
\end{aligned}
$$

where by (3.4), $\Psi\left(\rho_{i}^{-1}\right)>0$ satisfies

$$
\Psi\left(\rho_{i}^{-1}\right):=\max _{\alpha=1, \cdots, n}\left(1-L_{\alpha}^{-2} f_{B\left(x_{0}, 3 \rho_{i}\right)}\left|\nabla v_{\alpha}\right|^{2} \mathrm{~d} V_{g}\right) \rightarrow 0 \quad \text { as } \quad i \rightarrow \infty .
$$

Consequently, we have for each $\alpha=1, \cdots, n$,

$$
\rho_{i}^{2} f_{B\left(x_{0}, 2 \rho_{i}\right)}\left|\nabla \nabla u_{\alpha}\right|^{2} \mathrm{~d} V_{g} \leq C(M) L_{\alpha}^{2} \Psi\left(\rho_{i}^{-1}\right) .
$$


This estimate, together with the Poincaré inequality 2.1], controls the behavior of the average pull-back measure density on large scales:

$$
\begin{aligned}
& \left.f_{B\left(x_{0}, \rho_{i}\right)}|| \omega_{A_{\infty}}\right|^{2}-\operatorname{det} \Omega_{\rho_{i}}(\mathbf{v}) \mid \mathrm{d} V_{g} \\
\leq & \sum_{\sigma \in S_{n}} f_{B\left(x_{0}, \rho_{i}\right)}\left|\prod_{\alpha=1}^{n}\left\langle\nabla v_{\alpha}, \nabla v_{\sigma(\alpha)}\right\rangle-\prod_{\alpha=1}^{n} f_{B\left(x_{0}, \rho_{i}\right)}\left\langle\nabla v_{\alpha}, \nabla v_{\sigma(\alpha)}\right\rangle \mathrm{d} V_{g}\right| \mathrm{d} V_{g} \\
\leq & \sum_{\sigma \in S_{n}} \sum_{\alpha=1}^{n}\left(\prod_{\beta \neq \alpha} L_{\beta} L_{\sigma(\beta)}\right) f_{B\left(x_{0}, \rho_{i}\right)}\left|\left\langle\nabla v_{\alpha}, \nabla v_{\sigma(\alpha)}\right\rangle-f_{B\left(x_{0}, \rho_{i}\right)}\left\langle\nabla v_{\alpha}, \nabla v_{\sigma(\alpha)}\right\rangle \mathrm{d} V_{g}\right| \mathrm{d} V_{g},
\end{aligned}
$$

now for each $\alpha=1, \cdots, n$ and $\sigma \in S_{n}$ (the $n$-symmetric group), we apply the Poincaré inequality (2.1) and the Hölder inequality and (3.5) to see:

$$
\begin{aligned}
& f_{B\left(x_{0}, \rho_{i}\right)}\left|\left\langle\nabla v_{\alpha}, \nabla v_{\sigma(\alpha)}\right\rangle-f_{B\left(x_{0}, \rho_{i}\right)}\left\langle\nabla v_{\alpha}, \nabla v_{\sigma(\alpha)}\right\rangle \mathrm{d} V_{g}\right| \mathrm{d} V_{g} \\
\leq & C_{P} \rho f_{B\left(x_{0}, 2 \rho_{i}\right)}\left|\nabla\left\langle\nabla v_{\alpha}, \nabla v_{\sigma(\alpha)}\right\rangle\right| \mathrm{d} V_{g} \\
\leq & 2 C_{P}(m) C(M) L_{\alpha} L_{\sigma(\alpha)} \Psi\left(\rho_{i}^{-1}\right)^{\frac{1}{2}} .
\end{aligned}
$$

Consequently,

$$
\left.f_{B\left(x_{0}, \rho_{i}\right)}|| \omega_{A_{\infty}}\right|^{2}-\operatorname{det} \Omega_{\rho_{i}}(\mathbf{v}) \mid \mathrm{d} V_{g} \leq 2 C_{P}(m) C(M) n ! n\left(\prod_{\alpha=1}^{n} L_{\alpha}^{2}\right) \Psi\left(\rho_{i}^{-1}\right)^{\frac{1}{2}} .
$$

Combining (3.2), 3.3), 3.4) and (3.6), we see that

$$
\forall x \in M, \quad \lim _{i \rightarrow \infty} f_{B\left(x_{0}, \rho_{i}\right)}|\omega|^{2} \mathrm{~d} V_{g}=\prod_{\alpha=1}^{n} L_{\alpha}^{2} \geq|\omega|^{2}(x),
$$

where the last inequality stands as $\forall x \in M$,

$$
|\omega|^{2}(x)=\left|\omega_{A_{\infty}}\right|^{2}(x)=\left|\nabla v_{1} \wedge \cdots \wedge \nabla v_{n}\right|^{2}(x) \leq \prod_{\alpha=1}^{n}\left|\nabla v_{\alpha}\right|^{2}(x) .
$$

Since for every sequence $\rho_{i} \rightarrow \infty$, there is a subsequence for which (3.7) holds, we have finished proving 3.1 .

Rigidity. When $|\omega|\left(x_{0}\right)=\sup _{M}|\omega|$, we may renormalize $\tilde{v}_{\alpha}:=L_{\alpha}^{-1} v_{\alpha}$, so that $\left|\nabla \tilde{v}_{\alpha}\right|^{2} \leq 1$ and $\sup _{M}\left|\nabla \tilde{v}_{\alpha}\right|=1$ for $\alpha=1, \cdots, n$. By (3.3) and (3.4), we see $\operatorname{det} \Omega_{\rho_{i}}(\tilde{\mathbf{v}}) \rightarrow 1$. This fact, together with (3.6) and 3.7), ensure

$$
|\tilde{\omega}|\left(x_{0}\right)=\sup _{M}|\tilde{\omega}|=1 .
$$

On the other hand, since each $\left|\nabla \tilde{v}_{\alpha}\right|^{2}$ is bounded, non-negative and sub-harmonic, by Lemma 2.5 we have

$$
\lim _{\rho \rightarrow \infty} \int_{M}|\nabla \tilde{\mathbf{v}}|^{2} \mathrm{~d} \mu_{x_{0}}\left(\rho^{2}\right)=n
$$


By (1.6), the splitting error between the zero scale and the infinity scale is controlled as

$$
\begin{aligned}
|\nabla \tilde{\mathbf{v}}|^{2}\left(x_{0}\right) & \leq|\nabla \tilde{\mathbf{v}}|^{2}\left(x_{0}\right)+\int_{0}^{\infty} \int_{M}|\nabla \nabla \tilde{\mathbf{v}}|^{2} \mathrm{~d} \mu_{x_{0}}(t) \mathrm{d} t \\
& \leq \lim _{\rho \rightarrow \infty} \int_{M}|\nabla \tilde{\mathbf{v}}|^{2} \mathrm{~d} \mu_{x_{0}}\left(\rho^{2}\right) \\
& =n .
\end{aligned}
$$

However, applying the arithmetic mean - geometric mean inequality at $x_{0}$, we have

$$
n=n|\tilde{\omega}|^{\frac{2}{n}}\left(x_{0}\right) \leq|\nabla \tilde{\mathbf{v}}|^{2}\left(x_{0}\right),
$$

which, together with (3.8), ensures all inequalities there to be equalities; especially

$$
\int_{0}^{\infty} \int_{M}|\nabla \nabla \tilde{\mathbf{v}}|^{2} \mathrm{~d} \mu_{x_{0}}(t) \mathrm{d} t=0 \quad \Longrightarrow \quad \forall t>0, \quad \int_{M}|\nabla \nabla \tilde{\mathbf{v}}|^{2} \mathrm{~d} \mu_{x_{0}}(t)=0,
$$

whence the vanishing of $|\nabla \nabla \tilde{\mathbf{v}}|^{2}$ throughout $M$.

Since $\tilde{\mathbf{v}}$ is obtained from $\mathbf{u}$ by a linear transformation, this shows that $|\nabla \nabla \mathbf{u}|^{2} \equiv 0$ on $M$. The isometric splitting then follows easily from the vanishing of the splitting error of $\mathbf{u}$ and its non-degeneracy. For the details of the argument, see for instance [7].

Corollary 3.1. Suppose $(M, g)$ and $\mathbf{u}: M \rightarrow \mathbb{R}^{n}$ satisfy the same assumptions as in the strong maximum principle. We have $|\nabla \nabla \mathbf{u}| \equiv 0$ whenever one of the following conditions are satisfied:

(1) $|\nabla \omega| \equiv 0$

(2) $\Delta|\omega|^{2} \equiv 0$;

(3) $\Delta|\nabla \omega|^{2} \equiv 0$

Proof. Condition (1) implies that $|\omega| \equiv$ constant by a direct computation.

For Condition (2), first notice that $|\omega|^{2}=\left|\nabla u_{1} \wedge \nabla u_{2} \wedge \cdots \wedge \nabla u_{n}\right|^{2}$ is uniformly bounded throughout $M$, according to the control of $\mathbf{u}$ and Cheng-Yau gradient estimate [9] applied to each component of $\mathbf{u}$. Since Condition (2) says that $|\omega|^{2}$ is actually harmonic, applying Cheng-Yau's gradient estimate again to $|\omega|^{2}$ we immediately see that $|\omega|^{2}$ is a constant.

Since on $M$ any non-negative harmonic function is constant (Corollary 1 of [24]), Condition (3) implies that $|\nabla \omega|^{2} \equiv C \geq 0$. However, $|\nabla \omega|^{2} \leq C(m, n) L^{n-2}|\nabla \nabla \mathbf{u}|^{2}$ on $M$ and according to 3.5 ,

$$
\lim _{\rho \rightarrow \infty} f_{B(x, \rho)}|\nabla \nabla \mathbf{u}|^{2} \mathrm{~d} V=0
$$

Therefore, we have

$$
\begin{aligned}
\lim _{\rho \rightarrow \infty} f_{B(x, \rho)}|\nabla \omega|^{2} \mathrm{~d} V & \leq C(m, n) L^{n-2} \lim _{\rho \rightarrow \infty} f_{B(x, \rho)}|\nabla \nabla \mathbf{u}|^{2} \mathrm{~d} V \\
& =0,
\end{aligned}
$$

whence the constancy of $|\omega|$ throughout $M$.

\section{Weighted Poincaré inequality and applications}

In this section, we first set up the technical tools needed for proving Theorem 1.2 a Poincaré inequality on $M$ with respect to the heat measure $\mathrm{d} \mu_{x_{0}}(t)$. The long-time behavior of the heat solution, whose initial value is the pull-back measure density, is then studied following a similar strategy as in last section. 
4.1. Poincaré inequality weighted by the heat measure. We will control the weighted (by the heat measure) difference between a function $f \in L^{\infty}(M) \cap C^{1}(M)$ and its heat evolution, roughly by the heat evolution of its derivative (the "central part") and an error term (the "tail part").

The estimate of the "central part" is based on the heat kernel estimate of Li-Yau (Proposition 2.3), as well as Cheeger-Colding's segment inequality (Proposition 2.1). We begin with an elementary estimate of the distance to points on a minimal geodesic by that to the end points: Let $x, y \in M$ and $\gamma$ be a minimal geodesic connecting them. Since

$$
\forall s \in[0, d(x, y)], \quad d(x, \gamma(s))+d(y, \gamma(s))=d(x, y),
$$

we have

$$
\begin{aligned}
2 d\left(x_{0}, \gamma(s)\right) & \leq d\left(x_{0}, x\right)+d(x, \gamma(s))+d\left(x_{0}, y\right)+d(y, \gamma(s)) \\
& =d\left(x_{0}, x\right)+d\left(y_{0}, y\right)+d(x, y) \\
& \leq 2 d\left(x_{0}, x\right)+2 d\left(y_{0}, y\right)
\end{aligned}
$$

and thus

$$
\frac{1}{2} r^{2}(\gamma(s)) \leq r^{2}(x)+r^{2}(y)
$$

This gives, for almost every pair of $x, y \in M$, and $s \in[0, d(x, y)]$, that

$$
\begin{aligned}
|f(x)-f(y)| e^{-\frac{2}{9 t}\left(r^{2}(x)+r^{2}(y)\right)} & \leq \int_{0}^{d(x, y)}|\nabla f|(\gamma(s)) e^{-\frac{2}{9 t}\left(r^{2}(x)+r^{2}(y)\right)} \mathrm{d} s \\
& \leq \int_{0}^{d(x, y)}|\nabla f|(\gamma(s)) e^{-\frac{1}{9 t} r^{2}(\gamma(s))} \mathrm{d} s .
\end{aligned}
$$

Using this estimate, together with Proposition 2.3 and Proposition 2.1 we could control the "central part" as following:

$$
\begin{aligned}
& \int_{B\left(x_{0}, R\right)} \int_{B\left(x_{0}, R\right)}|f(x)-f(y)| \mathrm{d} \mu_{x_{0}}(x, t) \mathrm{d} \mu_{x_{0}}(y, t) \\
\leq & \frac{C_{2}(1 / 2)^{2}}{\left|B\left(x_{0}, \sqrt{t}\right)\right|^{2}} \int_{B\left(x_{0}, R\right)} \int_{B\left(x_{0}, R\right)}|f(x)-f(y)| e^{-\frac{2}{9 t}\left(r^{2}(x)+r^{2}(y)\right)} \mathrm{d} V_{g}(x) \mathrm{d} V_{g}(y) \\
\leq & \frac{C_{2}(1 / 2)^{2}}{\left|B\left(x_{0}, \sqrt{t}\right)\right|^{2}} \int_{B\left(x_{0}, R\right)} \int_{B\left(x_{0}, R\right)}\left(\int_{0}^{d(x, y)}|\nabla f|(\gamma(s)) e^{-\frac{1}{y} r^{2}(\gamma(s))} \mathrm{d} s\right) \mathrm{d} V_{g}(x) \mathrm{d} V_{g}(y) \\
\leq & C_{C C}(m) C_{2}(1 / 2)^{2} \frac{\left|B\left(x_{0}, 2 R\right)\right|}{\left|B\left(x_{0}, \sqrt{t}\right)\right|^{2}}(2 R) \int_{B\left(x_{0}, 2 R\right)}|\nabla f|(x) e^{-\frac{1}{9 t} r^{2}(x)} \mathrm{d} V_{g}(x) \\
\leq & C_{C C}(m) C_{2}(1 / 2)^{2} C_{1}(1) 3^{\frac{m}{2}} \frac{\left|B\left(x_{0}, 2 R\right)\right|}{\left|B\left(x_{0}, \sqrt{t}\right)\right|}(2 R) \int_{B\left(x_{0}, 2 R\right)}|\nabla f| \mathrm{d} \mu_{x_{0}}(3 t) .
\end{aligned}
$$

On the other hand, we could estimate the "tail part" of the heat measure for $R \geq \sqrt{t}$. Using the heat kernel upper bound and integrating radially, we have

$$
\begin{aligned}
\int_{M \backslash B\left(x_{0}, R\right)} 1 \mathrm{~d} \mu_{x_{0}}(t) & \leq \frac{C_{2}(1 / 2)}{\left|B\left(x_{0}, \sqrt{t}\right)\right|} \int_{M \backslash B\left(x_{0}, \sqrt{t}\right)} e^{-\frac{2 r^{2}(x)}{9 t}} \mathrm{~d} V_{g}(x) \\
& =\frac{C_{2}(1 / 2)}{\left|B\left(x_{0}, \sqrt{t}\right)\right|} \int_{R}^{\infty} e^{-\frac{2 r^{2}}{9 t}}\left|\partial B\left(x_{0}, r\right)\right| \mathrm{d} r .
\end{aligned}
$$

Since $M$ is complete and $\mathcal{R} c_{g} \geq 0$, by the Bishop-Gromov volume comparison, we have

$$
\left|\partial B\left(x_{0}, r\right)\right| \leq m r^{-1}\left|B\left(x_{0}, r\right)\right| \leq m r^{m-1} t^{-\frac{m}{2}}\left|B\left(x_{0}, \sqrt{t}\right)\right|,
$$


and thus

$$
\begin{aligned}
\int_{M \backslash B\left(x_{0}, R\right)} 1 \mathrm{~d} \mu_{x_{0}}(t) & \leq C_{2}(1 / 2) m \int_{R}^{\infty} e^{-\frac{2 r^{2}}{9 t}} r^{m-1} t^{-\frac{m}{2}} \mathrm{~d} r \\
& =C_{2}(1 / 2) m \int_{\frac{R}{\sqrt{t}}}^{\infty} e^{-\frac{2 s^{2}}{9}} s^{m-1} \mathrm{~d} s .
\end{aligned}
$$

Denoting

$$
\Psi_{2}(\sqrt{t} / R \mid m):=C_{2}(1 / 2) m \int_{\frac{R}{\sqrt{t}}}^{\infty} e^{-\frac{2 s^{2}}{9}} s^{m-1} \mathrm{~d} s
$$

we clearly see that

$$
\lim _{\frac{R}{\sqrt{t}} \rightarrow \infty} \Psi_{2}(\sqrt{t} / R \mid m)=0 .
$$

Combining the above estimates, we have the following Poincare inequality:

Lemma 4.1 (Weighted Poincaré inequality). Let $f$ be a bounded function on M. Suppose $|f| \leq L$ and $\int_{M}|\nabla f| d \mu_{x_{0}}(t)$ is defined for $t>0$. Then for $t>0$ and $R \geq \sqrt{t}$ we have

$$
\int_{M}\left|f-\int_{M} f d \mu_{x_{0}}(t)\right| d \mu_{x_{0}}(t) \leq C_{W P} \frac{\left|B\left(x_{0}, R\right)\right|}{\left|B\left(x_{0}, \sqrt{t}\right)\right|} R \int_{M}|\nabla f| d \mu_{x_{0}}(3 t)+\Psi_{W P}(\sqrt{t} / R \mid m) L,
$$

with $C_{W P}:=C_{C C}(m) C_{2}(1 / 2)^{2} C_{1}(1) 6^{m}$ being a dimensional constant and $\Psi_{W P}:=6 \Psi_{2}$.

Proof. Since $\int_{M} 1 \mathrm{~d} \mu_{x_{0}}(t)=1$, we see

$$
\begin{aligned}
& \int_{M}\left|f-\int_{M} f \mathrm{~d} \mu_{x_{0}}(t)\right| \mathrm{d} \mu_{x_{0}}(t) \\
\leq & \int_{B\left(x_{0}, R\right)} \int_{B\left(x_{0}, R\right)}|f(x)-f(y)| \mathrm{d} \mu_{x_{0}}(x, t) \mathrm{d} \mu_{x_{0}}(y, t)+3 \int_{M \backslash B\left(x_{0}, R\right)} 2 L \mathrm{~d} \mu_{x_{0}}(t),
\end{aligned}
$$

then the conclusion follows easily from the previous estimates (4.1), (4.2) and (4.3) .

4.2. Large-time evolution of the pull-back measure density by the heat equation. In this sub-section we prove Theorem 1.2. The proof follows in the same way as that of Theorem 1.1. However, due to the complication in the weighted Poincaré inequality (Lemma 4.1), for any sequence $t_{i} \rightarrow \infty$, we need to make a careful selection of the subsequences.

We will also need Li-Yau's Harnack inequality for the heat kernel (Proposition 2.4), which especially implies the following estimate: If $H_{x_{0}}(x, t)$ is the fundamental solution to the heat equation with initial value being the Delta function at $x_{0} \in M$, then for any $t_{2}>t_{1}>0$

$$
H_{x_{0}}\left(x, t_{1}\right) \leq\left(\frac{t_{2}}{t_{1}}\right)^{\frac{3 m}{4}} H_{x_{0}}\left(x, t_{2}\right)
$$

Proof of Theorem 1.2 In view of the proof of Theorem 1.1 we only need to show that for any $t_{i} \rightarrow \infty$, there is a subsequence $t_{i_{j}}$ such that

$$
\forall x \in M, \quad \lim _{j \rightarrow \infty} \int_{M}|\omega|^{2} \mathrm{~d} \mu_{x_{0}}\left(t_{i_{j}}\right) \geq|\omega|^{2}(x) .
$$

In a similar way as proving Theorem 1.1 we pick $t_{i} \rightarrow \infty$ and consider special orthogonal matrices $\bar{A}_{i}$ that diagonalize $\bar{\Omega}_{t_{i}}(\mathbf{u}):=\left[\int_{M} E_{\alpha \beta}(\mathbf{u}) \mathrm{d} \mu_{x_{0}}\left(t_{i}\right)\right]$. We then have some limiting 
$\bar{A}_{\infty} \in S O(n)$ to which a subsequence of $\bar{A}_{i}$ converges. For $\overline{\mathbf{v}}:=\bar{A}_{\infty} \mathbf{u}$, by the $S O(n)-$ invariance we have

$$
\begin{aligned}
& \operatorname{det} \bar{\Omega}_{t_{i}}(\mathbf{u})=\operatorname{det} \bar{\Omega}_{t_{i}}\left(\bar{A}_{i} \mathbf{u}\right)=\operatorname{det} \bar{\Omega}_{t_{i}}(\overline{\mathbf{v}}), \\
& \text { and }|\omega| \equiv\left|\omega_{\bar{A}_{i}}\right| \equiv\left|\bar{A}_{\infty} \omega\right| \quad \text { on } M .
\end{aligned}
$$

Moreover, invoking Lemma 2.5, we have

$$
\begin{aligned}
& \lim _{i \rightarrow \infty} \int_{M}\left|\nabla \bar{v}_{\alpha}\right|^{2} \mathrm{~d} \mu_{x_{0}}\left(t_{i}\right)=\sup _{M}\left|\nabla \bar{v}_{\alpha}\right|^{2}=: \bar{L}_{\alpha}^{2}, \\
& \text { and } \lim _{i \rightarrow \infty} \operatorname{det} \bar{\Omega}_{t_{i}}(\mathbf{u})=\prod_{\alpha=1}^{n} \bar{L}_{\alpha}^{2} .
\end{aligned}
$$

As made clear in the proof of Theorem 1.1 the key step is to obtain a heat measure version of (3.6). This will be our focus of the rest of the proof.

By (1.6) and Lemma2.5, we have for any $t>0$ and $\alpha=1, \cdots, n$,

$$
\begin{aligned}
2 \int_{3 t}^{4 t} \int_{M}\left|\nabla \nabla \bar{v}_{\alpha}\right|^{2} \mathrm{~d} \mu_{x_{0}}(s) \mathrm{d} s & \leq \int_{M}\left|\nabla \bar{v}_{\alpha}\right|^{2} \mathrm{~d} \mu_{x_{0}}(4 t)-\int_{M}\left|\nabla \bar{v}_{\alpha}\right|^{2} \mathrm{~d} \mu_{x_{0}}(3 t) \\
& \leq \Psi_{3}\left(t^{-\frac{1}{2}} \mid m\right) \bar{L}_{\alpha}^{2},
\end{aligned}
$$

with $\Psi_{3}\left(t^{-\frac{1}{2}} \mid m\right) \rightarrow 0$ as $t \rightarrow \infty$. So for some $\bar{t} \in[3 t, 4 t]$, we have

$$
2 t \int_{M}\left|\nabla \nabla \bar{v}_{\alpha}\right|^{2} \mathrm{~d} \mu_{x_{0}}(\bar{t}) \leq \Psi_{3}\left(t^{-\frac{1}{2}} \mid m\right) \bar{L}_{\alpha}^{2},
$$

and by Li-Yau's Harnack inequality 4.5], we have

$$
2 t \int_{M}\left|\nabla \nabla v_{\alpha}\right|^{2} \mathrm{~d} \mu_{x_{0}}(3 t) \leq\left(\frac{4}{3}\right)^{\frac{3 m}{4}} \Psi_{3}\left(t^{-\frac{1}{2}} \mid m\right) \bar{L}_{\alpha}^{2} .
$$

For each positive integer $j$, let $i_{j}$ be the first index so that $\forall i \geq i_{j}$,

$$
2 t_{i} \int_{M}\left|\nabla \nabla v_{\alpha}\right|^{2} \mathrm{~d} \mu_{x_{0}}\left(3 t_{i}\right) \leq j^{-2 m-3} \bar{L}_{\alpha}^{2}
$$

We could now estimate as before:

$$
\begin{aligned}
& \left.\int_{M}|| \omega_{\bar{A}_{\infty}}\right|^{2}-\operatorname{det} \bar{\Omega}_{t_{i_{j}}}(\mathbf{v}) \mid \mathrm{d} \mu_{x_{0}}\left(t_{i_{j}}\right) \\
\leq & \sum_{\sigma \in S_{k}} \sum_{\alpha=1}^{n}\left(\prod_{\beta \neq \alpha} \bar{L}_{\beta} \bar{L}_{\sigma(\beta)}\right) \int_{M}\left|\left\langle\nabla \bar{v}_{\alpha}, \nabla \bar{v}_{\sigma(\alpha)}\right\rangle-\int_{M}\left\langle\nabla \bar{v}_{\alpha}, \nabla \bar{v}_{\sigma(\alpha)}\right\rangle \mathrm{d} \mu_{x_{0}}\left(t_{i_{j}}\right)\right| \mathrm{d} \mu_{x_{0}}\left(t_{i_{j}}\right) .
\end{aligned}
$$

For each $\alpha=1, \cdots, n$ and $\sigma \in S_{n}$, we now apply the weighted Poincaré inequality (Lemma4.1) with the choice of $R_{j}:=j \sqrt{t_{t_{j}}}$, the Hölder inequality and (4.6) to see:

$$
\begin{aligned}
& \int_{M}\left|\left\langle\nabla \bar{v}_{\alpha}, \nabla \bar{v}_{\sigma(\alpha)}\right\rangle-\int_{M}\left\langle\nabla \bar{v}_{\alpha}, \nabla \bar{v}_{\sigma(\alpha)}\right\rangle \mathrm{d} \mu_{x_{0}}\left(t_{i}\right)\right| \mathrm{d} \mu_{x_{0}}\left(t_{i}\right) \\
\leq & C_{W P} j^{m+1} \sqrt{t_{i_{j}}} \int_{M}\left|\nabla\left\langle\nabla v_{\alpha}, \nabla v_{\sigma(\alpha)}\right\rangle\right| \mathrm{d} \mu_{x_{0}}\left(3 t_{i_{j}}\right)+\Psi_{W P}\left(j^{-1} \mid m\right) \bar{L}_{\alpha} \bar{L}_{\sigma(\alpha)} \\
\leq & 2 C_{W P} j^{-\frac{1}{2}} \bar{L}_{\alpha} \bar{L}_{\sigma(\alpha)}+\Psi_{W P}\left(j^{-1} \mid m\right) \bar{L}_{\alpha} \bar{L}_{\sigma(\alpha)} .
\end{aligned}
$$

Therefore as $j \rightarrow \infty$,

$$
\left.\int_{M}|| \omega_{\bar{A}_{\infty}}\right|^{2}-\operatorname{det} \bar{\Omega}_{t_{i_{j}}}(\mathbf{v}) \mid \mathrm{d} \mu_{x_{0}}\left(t_{i_{j}}\right) \leq\left(C_{W P} j^{-\frac{1}{2}}+\Psi_{W P}\left(j^{-1} \mid m\right)\right) \prod_{\alpha=1}^{n} \bar{L}_{\alpha}^{2} \rightarrow 0 .
$$


This is the heat measure analogue of (3.6) and it suffices to conclude the proof as argued before.

\section{Discussion}

5.1. Sub-harmonicity of the pull-back energy density. Continuing with the discussion and notations in the introduction, we recall that we have found $|\omega|^{2}$, as a bounded, nonnegative function on $M$, but is not necessarily sub-harmonic. However, there has not been any non-trivial example where the associated pull-back density of a vector valued harmonic map is shown not to be sub-harmonic. Notice that any meaningful example should be considered on a manifold which is non-parabolic (say, the volume of radius $r$ geodesic ball grows at least $\approx r^{3}$ ) and with vanishing volume ratio at infinity.

If it were shown that $\Delta|\omega|^{2} \geq 0$, then we would not need Theorem 1.2 to conclude (1.8). However, the following computation indicates that one should hardly expect $|\omega|^{2}$ to be sub-harmonic: Recall that $E_{\alpha \beta}=\left\langle\nabla u_{\alpha}, \nabla u_{\beta}\right\rangle$ and $\mathbb{E}=\left[E_{\alpha \beta}\right]$, a non-negative definite matrix valued function on $M$. Then $|\omega|^{2}=\operatorname{det} \mathbb{E}$, and

$$
\begin{aligned}
\Delta \operatorname{det} \mathbb{E} & =\sum_{\alpha, \beta=1}^{n}(-1)^{\alpha+\beta} \nabla \cdot\left(\operatorname{det} \mathbb{E}_{\alpha ; \beta}^{*} \nabla E_{\alpha \beta}\right) \\
& =\sum_{\alpha, \beta=1}^{n}(-1)^{\alpha+\beta}\left(\operatorname{det} \mathbb{E}_{\alpha ; \beta}^{*} \Delta E_{\alpha \beta}+\sum_{\gamma \neq \alpha, \delta \neq \beta}(-1)^{\gamma+\delta} \operatorname{det} \mathbb{E}_{\alpha \gamma ; \beta \delta}^{*}\left\langle\nabla E_{\gamma \delta}, \nabla E_{\alpha \beta}\right\rangle\right) \\
& =\sum_{\alpha, \beta=1}^{n} \sum_{\gamma \neq \alpha, \delta \neq \beta}(-1)^{\alpha+\beta+\gamma+\delta} \operatorname{det} \mathbb{E}_{\alpha \gamma ; \beta \delta}^{*}\left(E_{\gamma \delta} \Delta E_{\alpha \beta}+\left\langle\nabla E_{\gamma \delta}, \nabla E_{\alpha \beta}\right\rangle\right),
\end{aligned}
$$

where we use $\mathbb{E}_{\alpha_{1} \cdots \alpha_{k} ; \beta_{1} \cdots \beta_{k}}^{*}$ to denote the $(n-k, n-k)$-matrix obtained by deleting row $\alpha_{1}, \ldots, \alpha_{k}$ and column $\beta_{1}, \ldots, \beta_{k}$ from $\mathbb{E}$.

5.2. Directions of future research. The results in this paper leave the following directions open for further investigation:

(1) For a vector valued harmonic map u : $M^{m} \rightarrow \mathbb{R}^{n}(2<n \leq m)$, we consider the matrix $\mathbb{E}$ and denote the $k^{\text {th }}$-symmetric polynomial of the eigenvalues of $\mathbb{E}$ by $\sigma_{k}(k=1, \cdots, n)$. Suppose $\left(M^{m}, g\right)$ is complete, non-compact with non-negative Ricci curvature, and $\mathbf{u}$ is of at most linear growth, then Li's identities say that

$$
\lim _{\rho \rightarrow \infty} f_{B\left(x_{0}, \rho\right)} \sigma_{1} \mathrm{~d} V_{g}=\sup _{M} \sigma_{1}=\lim _{t \rightarrow \infty} \int_{M} \sigma_{1} \mathrm{~d} \mu_{x_{0}}(t) ;
$$

while Theorem 1.1 and Theorem 1.2 together tell that

$$
\lim _{\rho \rightarrow \infty} f_{B\left(x_{0}, \rho\right)} \sigma_{n} \mathrm{~d} V_{g}=\sup _{M} \sigma_{n}=\lim _{t \rightarrow \infty} \int_{M} \sigma_{n} \mathrm{~d} \mu_{x_{0}}(t) .
$$

It is therefore interesting to ask whether such identities hold for the symmetric polynomials in between, i.e. for $\sigma_{k}$ with $2 \leq k \leq n-1$, and whether achieving a global maximum of any of such $\sigma_{k}$ 's will induce isometric splitting of $M$.

(2) On the other direction, it is interesting to consider more general harmonic maps $\mathbf{u}:\left(M^{m}, g\right) \rightarrow\left(N^{n}, h\right)$ into a Cartan-Hardamad manifold $N$ (i.e. $(N, h)$ has nonpositive sectional curvature everywhere). Assume that $\left(M^{m}, g\right)$ is complete, noncompact and has non-negative Ricci curvature, then by the Weitzenböck formula, 
we have

$$
\begin{aligned}
\Delta|\nabla \mathbf{u}|^{2} & =2|\nabla \nabla \mathbf{u}|^{2}+2 \sum_{\alpha, \beta=1}^{m} \operatorname{Tr}_{h}\left(\mathcal{R} c_{\alpha \beta}^{M} \nabla_{\alpha} \mathbf{u} \nabla_{\beta} \mathbf{u}\right)-\sum_{\alpha, \beta=1}^{m}\left(\mathbf{u}^{*} \mathcal{R} m^{N}\right)_{\alpha \beta \beta \alpha} \\
& \geq 2|\nabla \nabla \mathbf{u}|^{2} .
\end{aligned}
$$

It is clear that if $\mathbf{u}$ has uniformly bounded energy density, then same argument as before (see Lemma2.5) gives

$$
\lim _{t \rightarrow \infty} \int_{M}|\nabla \mathbf{u}|^{2} \mathrm{~d} \mu_{x_{0}}(t)=\sup _{M}|\nabla \mathbf{u}|^{2} .
$$

Thus if $|\nabla \mathbf{u}|^{2}$ achieves the global maximum, then $|\nabla \nabla \mathbf{u}| \equiv 0$, so $N$ has to be the $n$ dimensional Euclidean space and $M$ isometrically splits $\mathbb{R}^{n}$. Again, it is interesting to see if such maximum principle still holds for the corresponding $\sigma_{k}$ for $k>1$, i.e. if any $\sigma_{k}$ sees a global maximum on $M$, then both $N \equiv \mathbb{R}^{n}$ and $M \equiv M^{\prime} \times \mathbb{R}^{n}$. See [19] for more information in this direction.

\section{REFERENCES}

[1] Jeff Cheeger, Degeneration of Riemannian metrics under Ricci curvature bounds (Lezioni Fermiane), Scuola Normale Superiore, Pisa (2001).

[2] Jeff Cheeger and Tobias Colding, Lower bounds on Ricci curvature and the almost rigidity of warped products, Ann. of Math. 144 (1996), 189-237.

[3] Jeff Cheeger and Tobias Colding, On the structure of spaces with Ricci curvature bounded below. I, J. Diff. Geom. 46 (1997), 406-480.

[4] Jeff Cheeger and Tobias Colding, On the structure of spaces with Ricci curvature bounded below. II, $J$. Diff. Geom. 54 (2000), 1-35.

[5] Jeff Cheeger and Tobias Colding, On the structure of spaces with Ricci curvature bounded below. III, J. Diff. Geom. 54 (2000), 37-74.

[6] Jeff Cheeger, Tobias Colding and William Minicozzi II, Linear growth harmonic functions on complete manifolds with non-negative Ricci curvature, Geom. Func. Anal. 6 (1995), 948-954.

[7] Jeff Cheeger and Detlef Gromoll, The splitting theorem for manifolds of nonnegative Ricci curvature, $J$. Diff. Geom. 6 (1971), 119-128.

[8] Jeff Cheeger and Aaron Naber, Regularity of Einstein manifolds and the codimension 4 conjecture, Ann. of Math. 182 (2015), 1093-1165.

[9] Shiu-Yuen Cheng and Shing-Tung Yau, Differential equations on Riemannian manifolds and their geometric applications, Comm. Pure Appl. Math. 28 (1975), 333-354.

[10] Tobias Colding and William Minicozzi II, Harmonic functions with polynomial growth, J. Diff. Geom. 46 (1997), 1-77.

[11] Tobias Colding and William Minicozzi II, Harmonic functions on manifolds, Ann. of Math. 146 (1997), 725-747.

[12] Tobias Colding and Aaron Naber, Sharp Hölder continuity of tangent cones for spaces with a lower Ricci curvature bound and applications, Ann. of Math. 176 (2012), 1173-1229.

[13] Harold Donnelly and Charles Fefferman, Nodal domains and growth of harmonic functions on noncompact manifolds, J. Geom. Anal. 2 (1992), 79-93.

[14] Peter Li, Uniqueness of $L^{1}$ solutions for the Laplace equation and the heat equation on Riemannian manifolds, J. Diff. Geom. 20 (1984), 447-457.

[15] Peter Li, Large time behavior of the heat equation on complete manifolds with non-negative Ricci curvature, Ann. of Math. 124 (1986), 1-21.

[16] Peter Li and Luen-Fai Tam, Linear growth harmonic functions on a complete manifold, J. Diff. Geom. 29 (1989), 421-425.

[17] Peter Li and Luen-Fai Tam, Complete surfaces with finite total curvature, J. Diff. Geom. 33 (1991), 139168.

[18] Peter Li and Richard Schoen, $L^{p}$ and mean value properties of subharmonic functions on Riemannian manifolds, Acta Math. 153 (1984), 279-301.

[19] Peter Li and Jia-Ping Wang, Convex hull properties of harmonic maps, J. Diff. Geom. 48 (1998), 497-530. 
[20] Peter Li and Shing-Tung Yau, On the parabolic kernel of the Schrödinger operator, Acta. Math. 156 (1986), 153-201.

[21] Laurent Saloff-Coaste, A note on Poincaré, Sobolev and Harnack inequalities, Int. Math. Res. Not. 2 (1992), 27-38.

[22] Richard Schoen and Shing-Tung Yau, Lectures on differential geometry, International Press, Boston (1994).

[23] Guoyi Xu, Large time behavior of the heat kernel, J. Diff. Geom. 98 (2014), 467-528.

[24] Shing-Tung Yau, Harmonic functions on a complete Riemannian manifold, Comm. Pure Appl. Math. 28 (1975), 201-228

Shaosai Huang, Department of Mathematics, Stony Brook University, 100 Nicolls Road, Stony Brook, NY, 11794-3651, U.S.A.

E-mail address: shaosai. huang@stonybrook. edu

Bing Wang, Department of Mathematics, University of Wisconsin - Madison, 480 Lincoln Drive, Madison, WI, 53706, U.S.A.

E-mail address: bwang@math.wisc.edu 